\title{
Opposing roles of TCF7/LEF1 and TCF7L2 in cyclin D2 and Bmp4 expression and cardiomyocyte cell cycle control during late heart development
}

\author{
Bo $\mathrm{Ye}^{1} \cdot$ Liwen $\mathrm{Li}^{2} \cdot$ Haodong $\mathrm{Xu}^{3} \cdot$ Yiping $\mathrm{Chen}^{2} \cdot$ Faqian $\mathrm{Li} \mathbb{D}^{1,4}$
}

Received: 17 August 2018 / Revised: 14 January 2019 / Accepted: 23 January 2019 / Published online: 18 February 2019

(c) United States \& Canadian Academy of Pathology 2019

\begin{abstract}
Bone morphogenetic protein (BMP) and Wnt pathways regulate cell proliferation and differentiation, but how these two pathways interact and mediate their nuclear actions in the heart, especially during late cardiac development, remains poorly defined. T-cell factor (TCF) and lymphoid enhancer factor (LEF) family transcriptional factors, including Lefl, Tcf7, Tcf7ll, and $T c f 7 l 2$, are important nuclear mediators of canonical Wnt/ $\beta$-catenin signaling throughout cardiac development. We reveal that these TCF/LEF family members direct heart maturation through distinct temporal and spatial control. TCF7 and LEF1 decrease while TCF7L1 and TCF7L2 remain relatively stable during heart development. LEF1 is mainly expressed in mesenchymal cells in valvular regions. TCF7 and TCF7L1 are detected in the nucleus of mesothelial and endothelial cells, but not in cardiomyocytes or mesenchymal cells. $T c f 7 / 2$ is the primary TCF/LEF family member in cardiomyocytes and undergoes alternative splicing during heart development. A TCF7L2 intensity gradient opposite to that of $\beta$-catenin and cardiomyocyte proliferative activity is present in fetal hearts. Wnt activation by cardiac deletion of APC, a negative Wnt regulator, dramatically increases Cyclin $D 2$ and Bmp4 expression. BMP signal transducing transcription factors, the mothers against decapentaplegic homologs (SMADs) are increasingly phosphorylated upon Wnt activation. LEF1/TCF7 displaces TCF7L2 and cooperates with pSMAD 1/5/8 in the regulatory elements of Cyclin D2 and Bmp4 promoters to promote $\beta$-catenin recruitment and transcriptional activation. Finally, we demonstrate that TCF7L2 is a transcriptional suppressor of Cyclin D2 and Bmp 4 in a cardiac cell line by overexpression and knockdown experiments.
\end{abstract}

Supplementary information The online version of this article (https:// doi.org/10.1038/s41374-019-0204-2) contains supplementary material, which is available to authorized users.

Faqian Li

lif@umn.edu

1 Department of Laboratory Medicine and Pathology, University of Minnesota, Room 293, Dwan Variety Club Cardiovascular Research Center, 425 E River Pkwy, Minneapolis, MN 55455, USA

2 Department of Cell and Molecular Biology, Tulane University, New Orleans, LA 70118, USA

3 Department of Pathology/Anatomic Pathology, University of Washington Medical Center, Seattle, WA 98195, USA

4 Lillehei Heart Institute and Masonic Cancer Center, University of Minnesota, Minneapolis, MN 55455, USA

\section{Introduction}

Global and local proliferative deficiency is the underlying mechanism of many congenital heart abnormalities such as ventricular hypoplasia and septal defects. The canonical Wnt/ $\beta$-catenin pathway has previously been shown to control cardiac proliferation and differentiation in several stages of cardiac development [1-3]. As a key component of this pathway, $\beta$-catenin expression level and sub-cellular location are tightly controlled by a multiprotein "destruction complex" that includes the tumor suppressors: axin and adenomatous polyposis coli (APC) and serine/threonine kinases: glycogen synthase kinase (GSK)-3 $\beta$ and casin kinase (CK) $1 / 2$ when Wnt signaling is off. Upon Wnt activation, this complex is disassembled and $\beta$-catenin becomes unphosphorylated and stabilized. Subsequently, $\beta$-catenin enters the nucleus and interacts with T-cell factor (TCF) and lymphocyte enhancer factor (LEF) family transcription factors to activate downstream Wnt target genes [4]. In simple organisms such as drosophila and C. elegans, 
there is only a single TCF/LEF gene [5]. During vertebrate evolution, the TCF/LEF family expanded to include four members: Lefl, Tcf7, Tcf7ll, and Tcf7l2. This allows functional diversification and specification in Wnt signal transduction [6]. Generally, TCF7L1 is considered a repressor of Wnt target genes [7], while TCF7 and LEF1 are frequently linked to Wnt target gene activation $[6,8]$. TCF7L2 can suppress or activate gene expressions dependent on its functional variants [8-10]. How individual TCF/ LEFs mediate Wnt signaling in the heart is poorly understood. Therefore, we examined the expression pattern of TCF/LEFs during cardiac development and investigated their roles in Wnt target gene expression. We found that TCF7L2 was the main TCF/LEF family member expressed in cardiomyocytes (CMs) in late embryonic development and suppressed Cyclin D2 and Bmp4 expression. Upon Wnt activation by $A p c$ deletion, there was a switch of TCF7L2 with TCF7 and LEF1 in the promoters of Cyclin D2 and Bmp4 to enhance $\beta$-catenin recruitment and promote gene transcription.

Canonical Wnt signaling can positive or negatively interact with bone morphogenetic protein (BMP) signaling $[11,12]$. During early cardiac development, similar cardiac defects have been reported in MesP1-Cre mediated deletion of Bmp receptor $1 a$ and $\beta$-catenin [13]. Furthermore, overexpression of a stabilized form of $\beta$-catenin enhances BMP production during early cardiac morphogenesis [13, 14]. However, opposite roles of Wnt and BMP pathways have been observed in colon crypt maturation and maintenance [12]. It is not clear how Wnt and BMP signals cooperate after cardiac chamber morphogenesis. Our results indicated that Wnt activation by cardiac Apc deletion promoted BMP signaling during late embryonic heart development.

\section{Materials and methods}

\section{Animals}

This study was carried out in strict accordance to the recommendations in the Guide for the Care and Use of Laboratory Animals (US Department of Health, Education, and Welfare, Department of Health and Human Services, NIH Publication 85-23). All procedures involving animals were approved by the Animal Care and Use Committee (IACUC) of the University of Minnesota, Minneapolis, Minnesota (Protocol 1503-32457A) and University of Rochester, Rochester, New York (Protocol 101341, UCAR2008-106R). All efforts were made to minimize suffering.

Mice with two loxP sites flanking exon 14 of $A p c$ $[15,16]$ were generated by Dr. Tetsuo Noda at The Cancer Institute of Japanese Foundation for Cancer Research
(Tokyo, Japan) and acquired from Dr. Bart O. Williams at the Van Andel Research lnstitute (Grand Rapids, MI, USA). Transgenic mice expressing Cre recombinase under the control of the $\alpha$-myosin heavy chain promoter $(\alpha M H C-C r e)$ were previously reported [17]. Homozygous Apc loxPfloxed $\left(A p c^{f / f}\right)$ mice were crossed with $\alpha M H C$-Cre mice to generate heterozygous loxP-floxed mice negative for $\alpha M H C$-Cre $\left(\mathrm{Apc}^{f /+} ; \alpha M H C-C r e-\right)$ and positive for $\alpha M H C$ $\mathrm{Cre}\left(\mathrm{Apc} \mathrm{f}^{f /+} ; \alpha M H C-\mathrm{Cre}+\right)$. CM-specific deletion of $\mathrm{Apc}$ in the hearts was achieved by crossing $\mathrm{Apc}^{\mathrm{f} /+}$; $\alpha M H C-\mathrm{Cr} e+$ mice to $A p c^{f f f}$ mice. This mating scheme was designed to generate $\alpha M H C$-Cre mice with two floxed alleles of $A p c$ (Apc ${ }^{f / f} ; \alpha M H C-C r e+$ further referred to as $\left.A p c \mathrm{cKO}\right)$. $\mathrm{Apc}^{f /+} ; \alpha M H C-C r e-, \quad A p c^{f / f} ; \alpha M H C-C r e-$ and $A p c^{f /+}$; $\alpha M H C-C r e+$ mice had identical morphology and Apcf/t; $\alpha M H C$-Cre- mice were used as wild type (WT) controls for all the experiments. Both WT and Apc cKO mice were also crossed into $\operatorname{Rosa}^{m T / m G}$ (membrane-Tomato/membraneGreen) fluorescent reporter line. Rosa ${ }^{m T / m G}$ mice contain two loxP sites flanking each side of the $\mathrm{mT}$ cassette. Mice express red fluorescence in all cell types and tissues before and green fluorescence after Cre-mediated recombination [18] (Fig. S1). Genomic DNA was isolated and purified with the DNeasy Blood \& Tissue Kit (Qiagen). Genotyping by PCR analysis was performed as follows: $\alpha M H C$-Cre transgene was amplified by PCR as described previously [19]. Analysis for Apc alleles was done by multiplex PCR using Apc-P3 primer 5'GTTCTGTATCATGGAAAGATAGGTGGTC-3', Apc-P4 primer 5'-CACTCAAAACGCTTTTGAGGGTTGATTC-3', Apc-P5 primer 5'-GAGTACGGGGTCTCTGTCTCAGTGAA-3', amplified as 314-bp targeted (P3 and P4), 258-bp deleted (P3 and P5), and 226-bp wild-type (P3 and P4) alleles, respectively $[15,16]$.

Mice were euthanized by deep anesthesia with continuous inhalation of $5 \%$ isoflurane until one minute after breathing stopped, then followed by cervical dislocation. The uterus was carefully dissected after euthanasia of pregnant dams. Fetuses of E13.5 and E17.5 were decapitated with surgical scissors and hearts were immediately fixed with $4 \%$ paraformaldehyde or snap frozen in liquid nitrogen and stored at $-80^{\circ} \mathrm{C}$ until analysis.

\section{RNA isolation, RT-PCR, quantitative RT-PCR, and RNA Sequencing}

Snap-frozen whole ventricles free of atrium and connective tissues from E13.5 and 17.5 embryos were homogenized and RNA was extracted using the TRIzol reagent (Invitrogen). One microgram of RNA was reverse transcribed to cDNA using a Quantitect Reverse Transcription Kit (Qiagen, Valencia, CA) in a reaction volume of $20 \mu$ l. For TaqMan ${ }^{\circledR}$ Assays, real-time quantitative PCR (qPCR) was performed with Applied Biosystems 7900HT Sequence Detection 
System in Genomics Research Center of University of Rochester on six pooled ventricles in duplicate with TaqMan ${ }^{\circledR}$ probes ordered from Applied Biosystems (Table S1). Data were normalized and standardized with SDS2.2 software provided by Applied System. For custom designed primers, real-time RT-PCR was performed on at least three different animals in triplicate in a $20 \mu \mathrm{l}$ reaction mixture with SYBR GreenER $^{\mathrm{TM}}$ qPCR SuperMix (BioRad Laboratories, Hercules, CA) on a CFX Connect ${ }^{\mathrm{TM}}$ Real-Time PCR Detection System (Table S2). The reaction conditions were as follows: $95^{\circ} \mathrm{C}$ for $15 \mathrm{~min}$, followed by 40 cycles of three steps consisting of denaturation at $94^{\circ} \mathrm{C}$ for $15 \mathrm{~s}$, primer annealing at $55^{\circ} \mathrm{C}$ for $20 \mathrm{~s}$, and primer extension at $72{ }^{\circ} \mathrm{C}$ for $20 \mathrm{~s}$. A melting curve analysis was performed from $65^{\circ} \mathrm{C}$ to $95^{\circ} \mathrm{C}$ in $0.3^{\circ} \mathrm{C}$ intervals. Gapdh was used to normalize differences in RNA input. Semi-quantitative RT-PCR reaction was performed using $1 \mu \mathrm{lDNA}$ in a total volume of $20 \mu \mathrm{l}$ containing $2 \mu \mathrm{l} 10 \times$ Taq buffer with $\mathrm{KCl}, 15 \mathrm{mM} \mathrm{MgCl}_{2}$ and $4 \mathrm{mM}$ dNTP (Thermo Fisher Scientific, Rockford, IL), 0.5 U Taq DNA Polymerase and $0.5 \mu \mathrm{M}$ of specific sense and antisense primers (Table S3) for each reaction.

RNA library preparation and sequence analysis were conducted by the Genomics Research Center at the University of Rochester. After determining of total RNA integrity using the Agilent 2100 Bioanalyzer, $1 \mu \mathrm{g}$ of high-quality total RNA was converted into a cDNA library using the mRNASeq sample preparation kit (Illumina) according to manufacturer's protocol. All reads were aligned to the mouse reference genome (NCBI 37, mm9) using TopHat. Transcript abundance was estimated as fragments per kilobase per million mapped reads (FPKM). Gene information including details of chromosomal position, start, end, gene product and Entrez ID, was obtained and reloaded to QIAGEN's Ingenuity Pathway Analysis (IPA). RNA sequencing data have been deposited in https://www.ncbi.nlm.nih.gov/sra/ PRJNA509930. Wnt//-catenin pathway related genes, including TCF/LEF family were extracted and analyzed by statistical software $R$.

\section{Histology and immunohistochemistry}

Embryonic hearts were fixed in 4\% paraformaldehyde for 30 min at room temperature and embedded in paraffin. Fourmicrometer sections were cut for hematoxylin and eosin or immunohistochemical staining as previously described [20]. Antigen retrieval was conducted in EDTA buffer (pH: 9.0) by heating to $99^{\circ} \mathrm{C}$ for $20 \mathrm{~min}$ with a DAKO PT Link PreTreatment Module Tissue Processor (Carpinteria, CA). Primary antibodies (Table S4) were applied to slides for overnight at $4{ }^{\circ} \mathrm{C}$. Immunohistochemical staining was performed using HistoMouse ${ }^{\mathrm{TM}}$-SP Kit (Invitrogen, Carlsbad, CA) according to manufacturer's instruction throughout this study.

\section{Immunofluorescent labeling and confocal microscopy}

Four percent paraformaldehyde-fixed hearts were washed with PBS and immersed in 30\% sucrose/PBS for $30 \mathrm{~min}$ to $1 \mathrm{~h}$ and then embedded in Tissue-Tek OCT compound (Sakura Finetek). Five-micrometer sections were blocked with $10 \%$ normal goat serum for $60 \mathrm{~min}$ and then incubated with indicated primary antibodies (Table S4) for overnight at $4{ }^{\circ} \mathrm{C}$. After washing six times with PBS, secondary antibody labeled with DyLight-555 or 488 was added. Nuclear counterstain was performed with $4^{\prime}, 6$-Diamidino2-Phenylindole (DAPI, Sigma-Aldrich, St. Louis, MO). Confocal images were collected with Olympus FV1000 confocal microscope (Olympus America Inc., Melville, NY) under uniform settings. For each antibody, staining was performed on at least three mice of each genotype.

\section{Quantification of TCF7L2 fluorescent intensity}

Five-micrometer frozen sections were co-stained with TCF7L2 and sarcomeric $\alpha$-actinin (Actinin) antibodies. Nuclei were visualized with DAPI counter staining. Ventricle images were collected by confocal microscopy at $\times 40$ magnification with a z-step of $0.55 \mu \mathrm{m}$ using an Olympus FV1000 confocal microscope. Five sequential images along the $\mathrm{z}$-axis were stacked to generate a $3 \mathrm{D}$ image for fluorescence intensity quantification by imageJ (Fiji). Ventricular walls of embryonic hearts were divided into three different zones as following: zone I, compact layer; zone II, outer trabecular layer from the junction of compact and trabecular layer up to $150 \mu \mathrm{m}$ from the epicardium; zone III, inner trabecular layer. The nuclear intensity of TCF7L2 was measured in DAPI stained CM nuclei of all Actinin positive regions within zone I, II, and III under $\times 40$ magnification respectively, and presented as the mean fluorescence intensity in pixels. (Fig. 2a).

\section{Western blot analyses}

Protein samples from E17.5 ventricles of WT and Apc cKO animals were analyzed by SDS-PAGE and immunoblotting with primary antibodies, followed by HRPconjugated goat secondary antibodies (Cell Signaling). The HRP signal was visualized by SuperSignal West Pico Chemiluminescent Substrate (Thermo Scientific) and the relative density of each band was quantified by densitometry.

\section{In situ hybridization}

Probe preparation and in situ hybridization were carried out as described previously with several modifications 
[21, 22]. In brief, antisense DIG (digoxigenin)-labelled RNA probes were transcribed in vitro from the plasmid with the cDNA encoding of murine BMP4 using the Ambion ${ }^{\circledR} \quad$ MAXIscript $^{\circledR}$ T7 Transcription Kit (Ambion, Waltham, MA, USA). E13.5 embryos were fixed with $4 \%$ PFA for overnight then embedded in Tissue-Tek OCT compound (Sakura Finetek). Tenmicrometer-thick cryosections were treated with proteinase $\mathrm{K}$, and then prehybridized in prehybridization buffer containing $50 \%$ formamide (Amresco, Solon, OH), $5 \times$ SSC (Invitrogen, USA), $50 \mu \mathrm{g} / \mathrm{mL}$ tRNA (Roche, Mannheim, Germany), $50 \mu \mathrm{g} / \mathrm{mL}$ heparin (Sigma-Aldrich, St. Louis, MO), 2\% blocking reagent (Sigma-Aldrich, St. Louis, MO), $0.1 \%$ CHAPS (Sigma-Aldrich, St. Louis, MO), $0.1 \%$ Tween 20 and $1 \mathrm{mM}$ EDTA at $70{ }^{\circ} \mathrm{C}$ for $2 \mathrm{~h}$. Hybridization was performed with DIG-labeled RNA probes for $16 \mathrm{~h}$. After incubating with anti-DIG alkaline phosphatase conjugate (Roche Diagnostics USA) overnight, hybridization signal was visualized by using the BCIP/NBT color development substrate (Promega, Madison, WI).

\section{Chromatin immunoprecipitation (ChIP)}

ChIP was conducted with Magna $\mathrm{ChIP}^{\mathrm{TM}} \mathrm{G}$ Chromatin Immunoprecipitation Kit (Millipore, Billerica, MA) as described previously [23]. Pooled ventricles from embryos at E17.5 were quickly washed three times with PBS and subjected to a cross-linking reaction with $1 \%$ formaldehyde for $12 \mathrm{~min}$ at room temperature. After quenching with $0.1 \mathrm{M}$ glycine, hearts were extracted in $1 \%$ SDS, $10 \mathrm{mM}$ EDTA, and $50 \mathrm{mM}$ Tris- $\mathrm{HCl}$, (pH 8.1) with shaking at $4{ }^{\circ} \mathrm{C}$ for $1 \mathrm{~h}$ and then sonicated to an average DNA size of 200-500 bp (assessed by agarose gel electrophoresis) using a Sonifier (Model 250, VWR Scientific, Danbury, USA) at $40 \%$ power output. The sonicated extracts were centrifuged and diluted with ChIP buffer (16.7 mM TrisHCl, pH 8.1, $167 \mathrm{mM} \mathrm{NaCl,} \mathrm{0.01 \%}$ SDS, $1.1 \%$ Triton X-100, 1.2 mM EDTA). Chromatin was incubated with the indicated antibodies (Table S4) and 20 $\mu \mathrm{L}$ Protein $\mathrm{G}$ magnetic beads for overnight with rotation at $4{ }^{\circ} \mathrm{C}$. An equal amount of mouse or rabbit $\mathrm{IgG}$ was used as a negative control following the same steps. The Protein $\mathrm{G}$ bead-antibody/chromatin complex was pelleted and washed extensively. After de-crosslinking at $62^{\circ} \mathrm{C}$ for $2 \mathrm{~h}$, the DNA was recovered and collected. The released DNA fragments were purified with columns and analyzed by PCR with the following primers that flank the TCF/LEF and SMAD binding sites in the Cyclin D2 and Bmp4 promoters (Fig. 5a). TCF/LEF binding site in D2 promoter $(-170$ to +124$)$, 5'-CGGGGATCGTGTTTGA AGTT-3' and 5'-AGAGAAGAGTGGAAGGTGGG-3'. SMAD binding site in D2 promoter ( -869 to -582$)$,
5'-CGAGCCATTTCCTAGAAAGC-3' and 5'-GGAGAG GAAGAGGAGGAAGG-3'. TCF/LEF binding site in Bmp4 promoter (-1087 to -767), 5'-GCAGCTTTCCC TTTAGCAGA-3' and 5'-CAGTGCCTTTTAGGCCATGT-3'. SMAD binding site in Bmp4 promoter $(-453$ to -180), 5'-CTGCTCACAGCCTGTTTCAA-3' and 5'-GACCACGTGCAAGAGGTACT-3'.

\section{Viral production and HL-1 cell infection}

Adenoviral vector containing a wild type full-length (long form) of $T c f 7 l 2$ was purchased from Vector BioLabs (Malvern, PA). The vector was propagated and amplified in $293 \mathrm{~T}$ cells according to standard protocols. The plaque assay was used to determine adenoviral titer. A shRNA construct targeting mouse Tcf7l2 was obtained from Sigma-Aldrich (St Louis, MO). The 21 bp sequence was 5'-GCTCCGAAAGTTTCCGAGATA-3'. Third-generation VSV-G pseudotyped high titers lentiviruses were generated by transient co-transfection of $293 T$ cells using Lenti-X ${ }^{\text {TM }}$ HTX Packaging System (Clontech) according to the manufacture's instruction. A control vector expressing GFP (pGIPZ) was used to monitor lentivirus infection efficiency. Lentiviruscontaining supernatant was collected, and the titer was determined by Lenti-X p24 rapid titer assay (Clontech). HL-1 atrial-derived CMs were seeded at a density of $1 \times$ $10^{5}$ cells/plate. Adenovirus or lentivirus supernatants were added to $10^{5}$ target cells (Opti-MEM 2\% FBS) at an estimated MOI of 50 in the presence of $10 \mu \mathrm{g} / \mathrm{ml}$ polybrene (Sigma) and incubated for $24 \mathrm{~h}$. Cells were then washed with and cultured in Claycomb medium $10 \%$ FBS (Sigma-Aldrich, F-2442). RNA was extracted $72 \mathrm{~h}$ after infection for real time RT-PCR.

\section{Statistics}

Fluorescent intensity and real time RT-PCR data were presented as mean \pm standard deviation (SD) of the mean. Means among groups were analyzed with SPSS 15.0 software (IBM Corporation, Armonk, NY). Student $t$ test was used for 2-group analysis with equal variances. If equal variance was not assumed, the Satterthwaite's approximate $t$ test was used for 2-group analysis. Oneway ANOVA was employed for multiple groups' comparison. Post hoc multiple comparisons were then performed to determine if a statistical significance was detected between groups. Fisher's least significant difference (LSD) was used to compare group means when equal variances were present. If equal variances were not assumed, differences between group means were calculated by Tamhane's T2 test. A $P$ value smaller than 0.05 was considered statistically significant. 
A

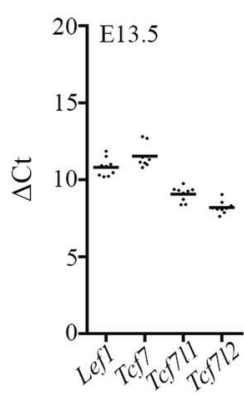

B

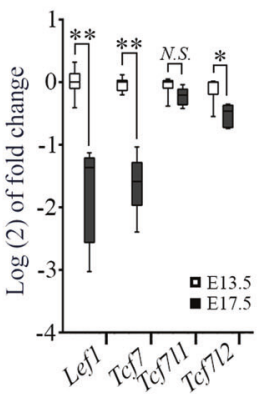

Color key and histogram
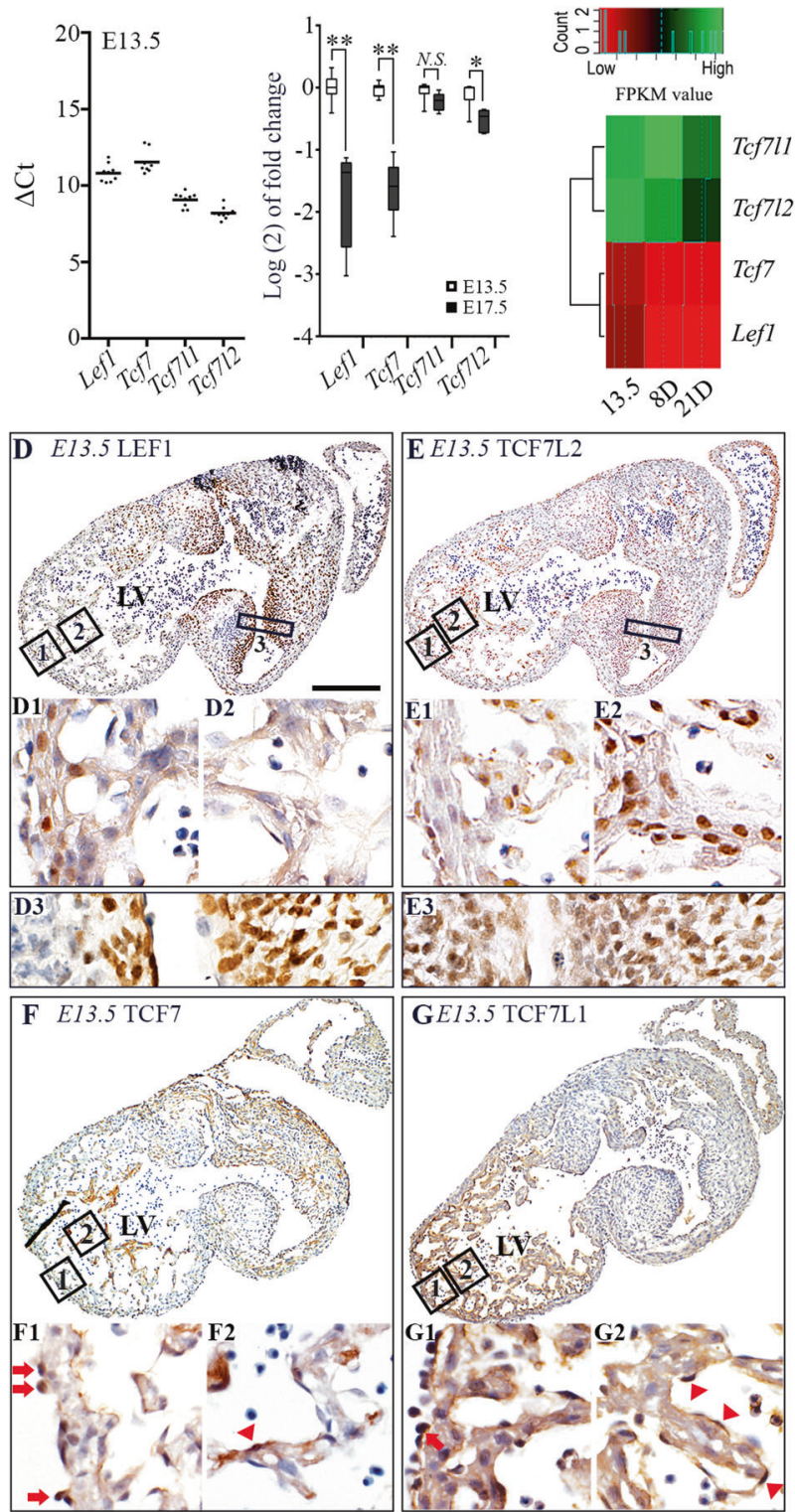

Fig. 1 TCF/LEFs expression in mouse hearts at E13.5 and E17.5. a Cardiac mRNA expressions of TcflLef family at E13.5 are represented as mean delta $\mathrm{Ct}(\Delta \mathrm{Ct})$ values normalized by Gapdh. b The $\log 2$ fold changes of $T c f / L e f$ mRNA levels at E17.5 versus E13.5 in murine hearts. c A heat map shows fragments per kilobase of transcript per million mapped reads (FPKM) for Tcf/Lef genes in murine hearts from E13.5 to postnatal day 21. d-g Expression of LEF1, TCF7, TCF7L1 and TCF7L2 in mouse hearts at E13.5 by immunohistochemistry. LEF1 shows clear and strong nuclear staining in cells of atrioventricular (AV) cushions and valves (D3). Scattered CMs showing moderate to weak nuclear staining for LEF1 are also observed in the compact (D1) and trabecular (D2) layers. Moderate nuclear staining of TCF7L2 is mainly observed in CMs of the trabecular layer (E2) and cells of AV cushions and valves (E3). CMs in the compact layer barely or weakly stain positive for TCF7L2 (E1). Nuclear TCF7 and TCF7L1 are not detected in CMs of both compact and trabecular layers $(\mathbf{f}-\mathbf{g})$ at E13.5 by immunohistochemistry. Epicardial (arrows) and endothelial cells (triangles) lining the outer and inner cardiac surfaces respectively are positive for TCF7 and TCF7L1. Scale bar $=200 \mu \mathrm{m}$. Data represent mean \pm SD. $N=4$ independent experiments

\section{Results}

\section{The expression of TCF/LEFs during heart development}

The overall expression pattern of TCF/LEFs in mice during development has been examined by in situ hybridization at mRNA levels (http://www.genepaint.org) [24, 25]. However, the detailed expressional changes of these factors in different regions and cell types during cardiac development are not clearly defined. We performed real-time RT-PCR and RNA sequencing to examine TCF/LEF gene expression. RNA sequencing data have been deposited in https://www.ncbi.nlm.nih. gov/sra/PRJNA509930. Tcf7l2 was the most abundant TCF/LEF transcripts in the murine heart at E13.5 by realtime RT-PCR (Fig. 1a) and the fragments per kilobase of transcript per million mapped reads (FPKM) of $T c f 7 l 2$ was also the highest among TCF/LEF family members in the heart at E13.5 by RNA sequencing (Fig. 1c). With immunohistochemical staining, mesenchymal cells in valvular regions had much stronger intensity of LEF1 than $\mathrm{CMs}$ in ventricles. CMs with weak to moderate nuclear staining for LEF1 were scattered in compact and trabecular layers (Fig. 1d). At E17.5, total Lef1 mRNA was dramatically decreased (Fig. 1b) and it was no longer detectable in $\mathrm{CMs}$ by immunofluorescence, although nuclear LEF1 signals were still observed in valvular regions (Fig. S2A). Tcf7 mRNA was the lowest among all four TCF/LEF members at E13.5 (Fig. 1a, c) and it further decreased at E17.5 (Fig. 1b). No TCF7 protein was detected by immunohistochemistry in CMs or mesenchymal cells in contrast to its nuclear positivity in mesothelial and endothelial cells lining the out and inner surfaces of the heart respectively (Fig. 1f and Fig. S2B). Tcf7l1 mRNA levels were relatively stable from E13.5 to E17.5 (Fig. 1b). Only weak cytoplasmic, but not nuclear, TCF7L1 signals were detected in CMs at E13.5 by immunochemistry. Interestingly, mesenchymal cells at valvular regions had no detectable TCF7L1 protein expression (Fig. 1g). By E17.5, CMs also had no detectable TCF7L1 protein. Tcf7l2 mRNA mildly decreased from E13.5 to E17.5, but was still relatively abundant in E17.5 murine hearts. By immunohistochemistry, both CMs and mesenchymal cells expressed nuclear Tcf712 (Fig. 1e). Endothelial cells and mesothelial cells were negative for TCF7L2. TCF7L2 was the only TCF/LEF member detected in CMs by immunohistochemistry at E17.5. Moreover, it had an interesting distribution gradient in CMs across the mouse ventricular wall. During mouse heart development, $T c f 7 l 2$ underwent alternative splicing and $T c f 7 l 2$ without exon 4 increased from E13.5 to postnatal day (D) 4 while constitutive exons 7 and 8 
remained relatively stable (Fig. S7). Additionally, neonatal hearts have the most abundant $T c f 7 l 2$ transcripts with exon 14.

\section{TCF7L2 ventricular intensity gradient at E13.5 and E17.5}

CMs show a proliferation gradient across the ventricular wall with the highest proliferation rate at the epicardial compact layer of embryonic hearts [26]. We previously demonstrated that the key Wnt signal transducer, $\beta$-catenin, formed a signaling intensity gradient which positively correlated with $\mathrm{CM}$ proliferative activity across the ventricular wall while the Wnt negative regulator, adenomatosis polyposis coli (APC), had a reverse distribution pattern at E13.5 and 17.5. Here, we have further demonstrated that CMs in the trabecular layer had stronger nuclear positivity for TCF7L2 than mesenchymal cells in the valvular regions. On the other hand, CMs in the compact layer had very weak nuclear staining for TCF7L2 (Fig. 2d). We quantified the nuclear TCF7L2 intensity of $\mathrm{CMs}$ in three zones from the epicardium to the endocardium: zone I, compact layer; zone II, outer trabecular layer from the junction of compact and trabecular layer up to $150 \mu \mathrm{m}$ from the epicardium; and zone III, inner trabecular layer (Fig. 2a). The nuclear TCF7L2 intensity of CMs gradually increased from zone I to III and reached its highest level at the innermost trabecular layer (Fig. 2b-d). Similarly, the percentage of TCF7L2 positive CMs increased from $6.8 \%$ in the zone I, $37.2 \%$ in the zone II to $73.3 \%$ in the zone III (Fig. 2c). Therefore, TCF7L2 had an intensity gradient similar to the distribution of APC, but opposite of nuclear $\beta$-catenin (Fig. S3) across the ventricular wall.

\section{Differential regulation of TCF/LEFs upon Wnt activation in the heart}

We previously showed that $\alpha M H C$-Cre could mediate Apc deletion in fetal CMs with a well-characterized mouse model containing LoxP sites flanked at the exon 14 of the Apc gene $\left(A p c^{f}\right)[15,16]$ to activate $\mathrm{Wnt}$ signaling. All genotypes were present at expected numbers (Table S5). Tomato reporter mice with $\operatorname{Ros}^{\mathrm{mT} / \mathrm{mG}}$ [18] also revealed excellent excision activity of $\alpha M H C$-Cre in both compact and trabecular layers at E13.5 and E17.5. Apc cKO mice had thick ventricles with increased CM proliferation and showed $\beta$-catenin accumulation and nuclear translocation in CMs [3]. To determine how TCF/LEFs are regulated in the heart by Wnt signaling, we used Apc cKO animal models to profile TCF/LEFs expression. At E13.5 and E17.5, Tcf7l2 mRNA was decreased by 15-20\% while Lefl and Tcf7 mRNAs were increased by 2.4-3.5 and 1.4-1.7-fold in Apc cKO mice compared to WT controls respectively. No obvious change in Tcf7ll mRNA was detected between $A p c$ cKO and WT littermates (Fig. 3a).
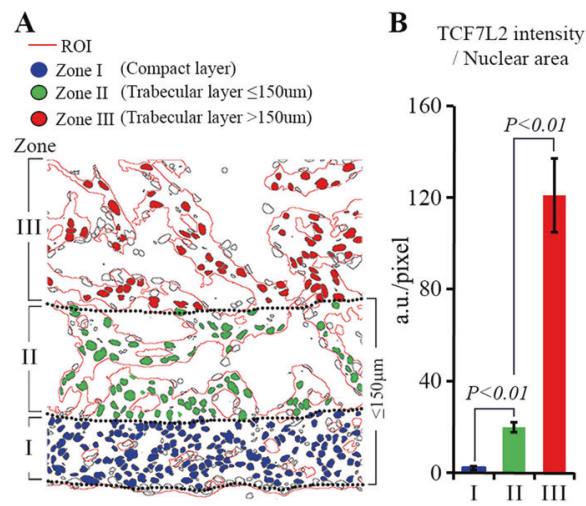

C ${ }_{\text {TCF7L2 }}+\mathrm{CMs}$
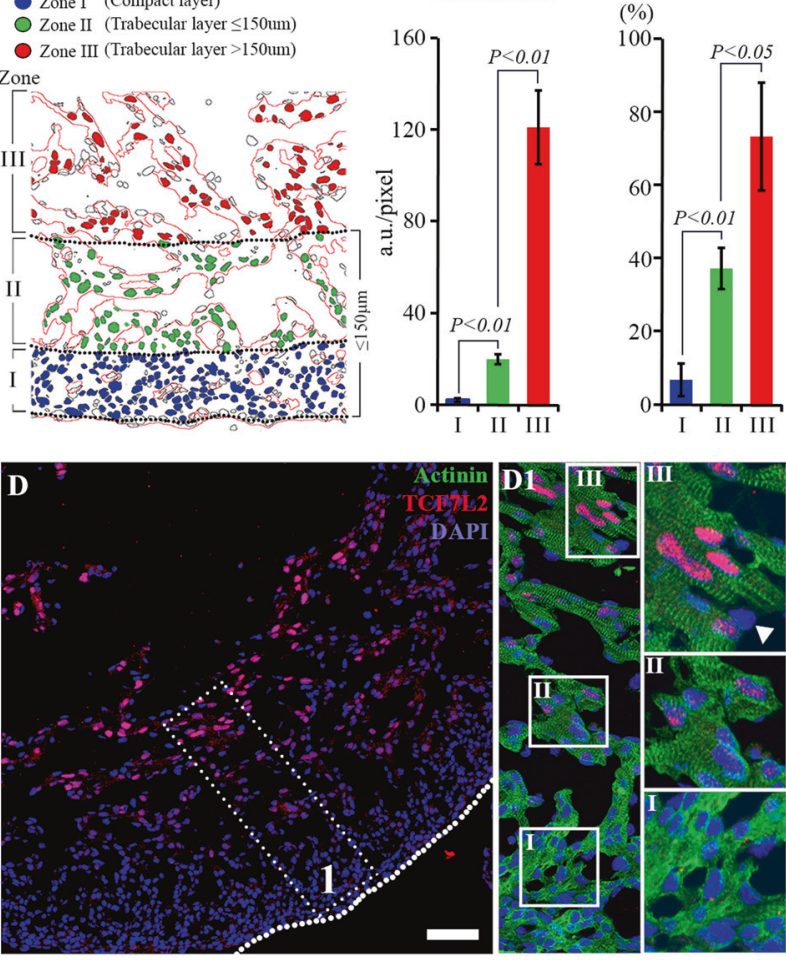

Fig. 2 TCF7L2 gradient across the ventricular wall of normal E13.5 embryos. a An illustration (a) shows how nuclear TCF7L2 intensity was measured from different areas of ventricle walls, including the compact layer (zone I), outer trabecular layer (zone II) and inner trabecular layer (zone III). Region of interests (ROI) are semiautomatically outlined by image $\mathrm{J}$ based on Actinin positive areas. Nuclei of CMs within ROIs are semi-automatically selected in different zones according to the DAPI staining. b, c Bar graphs demonstrate average nuclear TCF7L2 intensity (b) and the percentage of TCF7L2 positive CMs (c) in different zones across ventricular walls of E13.5. d Nuclear TCF7L2 signal is gradually increased from the outer compact layer to the inner trabecular layer forming an intensity gradient across the ventricle wall. Boxed region (D1) is magnified and showed with double staining for TCF7L2 and Actinin. TCF7L2 positive cells from zone I, II and III are mainly Actinin positive CMs. Endothelial cells are negative for TCF7L2 (triangles). Dotted lines show visceral pericardium and bar $=50 \mu \mathrm{m}$. Data represent mean \pm SD. $N=4$ independent experiments

The protein levels of full-length LEF1 $(\sim 58 \mathrm{KD})$ and TCF7 $(\sim 50 \mathrm{KD})$ were dramatically upregulated at E17.5 in Apc cKO ventricles by Western blotting (Fig. 3b, c). Immunofluorescent labeling and confocal microscopy revealed that the upregulation of LEF1 and TCF7 occurred exclusively in $\mathrm{CMs}$ and colocalized with nuclear $\beta$-catenin signals in $A p c$ deletion hearts at E17.5 (Fig. 3d-g). Conversely, full-length 75kDa TCF7L2 expression was reduced (Fig. 3b, c), but its gradient was still maintained in Apc cKO hearts (Fig. S4). Interestingly, the shorter full length $54 \mathrm{kDa}$ TCF7L2 is mildly increased (Fig. 3b). Thus, LEF1, TCF7, TCF7L1 and TCF7L2 responded differently to $\mathrm{Wnt} / \beta$-catenin pathway activation induced by $A p c$ deletion, indicating that they may 

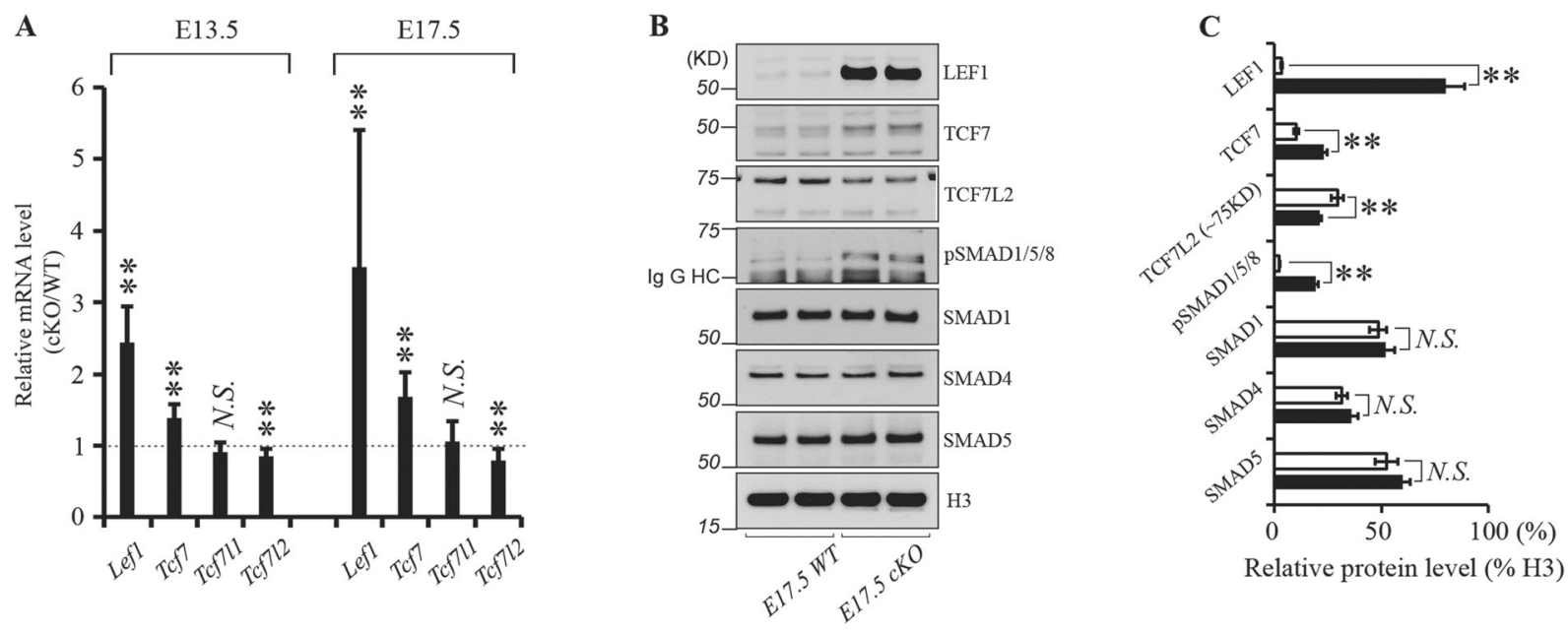

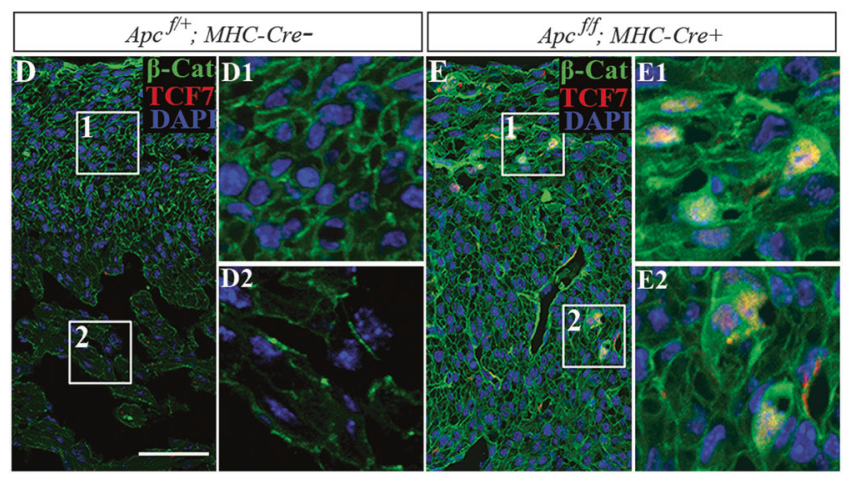

Fig. 3 Alteration of $\beta$-catenin partners by Apc deletion. a A bar graph shows the ratio of TcflLef mRNA expression levels of hearts between Apc knockout (KO) animal and their wild type (WT) siblings at E13.5 and E17.5 by q-PCR. b Upregulation LEF1 and TCF7, but downregulation of long form of TCF7L2 $(\sim 75 \mathrm{kDa})$ upon cardiac Apc deletion at E17.5 by western blot. Short form of TCF7L2 $(\sim 54 \mathrm{kDa})$ is mildly increased. pSMAD1/5/8 is also increased by ablation of Apc. Uncropped images of Western blots are displayed in Fig. S6. c Band intensities are quantified by ImageJ and normalized to Histone 3 .

have differential roles in transmitting $\mathrm{Wnt} / \beta$-catenin signal to the nucleus.

\section{Wnt activation promotes BMP signaling}

Canonical Wnt signaling has been shown to interact with several key developmental signaling pathways in mouse hearts $[13,14,27,28]$. To gain mechanistic insight into this pathway crosstalk, we screened transcriptional outputs of several signaling pathways in $A p c$ cKO hearts at E13.5 by real time qRT-PCR and found a significant increase in Bmp4 mRNA, but Bmp2, Bmp7 and Bmp10 were not affected by $A p c$ deletion (Fig. 4c). The upregulation of Bmp4 mRNA expression in CMs of E13.5 Apc cKO mice was further confirmed by in situ hybridization (Fig. 4d, e). Persistent elevation of Bmp4 mRNA levels was detected in $A p c$ deleted hearts at E17.5 (Fig. 4f). As nuclear transducers

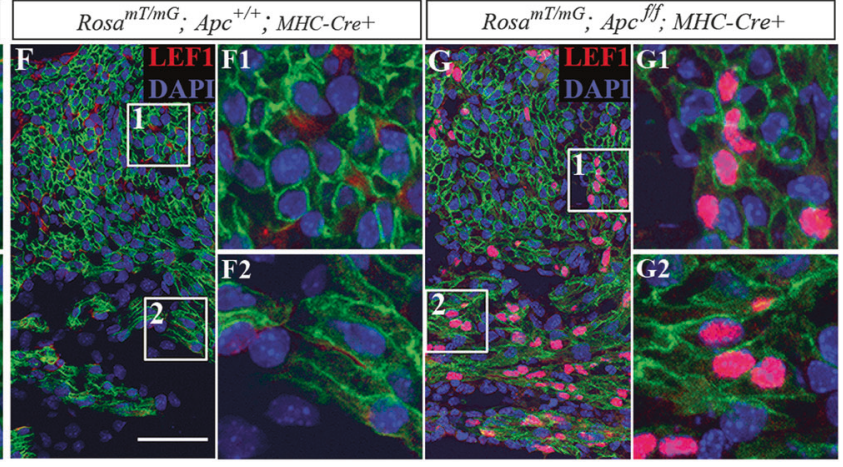

d, e TCF7 was not detected in WT (d), but was induced in Apc KO mice and colocalized with nuclear $\beta$-catenin ( $\beta$-Cat) in CMs (e) at E17.5. f, $\mathbf{g}$ Tomato hetero hearts revealed efficient conversion of membranous red (not shown well due to weaker signal than that of LEF1) to green fluorescence by $\alpha M H C$-Cre. LEF1 was not detected in WT (f), but was dramatically elevated in Apc KO mice (g) at E17.5. Scale bars $=50 \mu \mathrm{m}$. Data represent mean \pm SD. $N=4$ independent experiments

of BMP signaling, pSMAD1/5/8 were also increased by $A p c$ deletion (Fig. 3b, c). Although pSMAD1/5/8 were detected in both CMs and non-CMs in WT embryos at E13.5 and 17.5 by immunolabeling, pSMAD1/5/8 were increased only in CMs when $\beta$-catenin was stabilized in $A p c$ deficient hearts (Fig. 4a and Fig. S5).

\section{Wnt activation enhances target gene expression by switching TCF7L2 with TCF7 and LEF1 and recruiting pSMADs}

TCF/LEFs are sequence-specific transcription factors which exhibit remarkable amino-acid sequence conservation in the high-mobility group (HMG) DNA-binding domain and nuclear localization signal (NSL). TCF/LEFs bind to the consensus sequence WWCGGGTT and act as downstream nuclear effectors in the $\mathrm{Wnt} / \beta$-catenin pathway [6]. D-type 

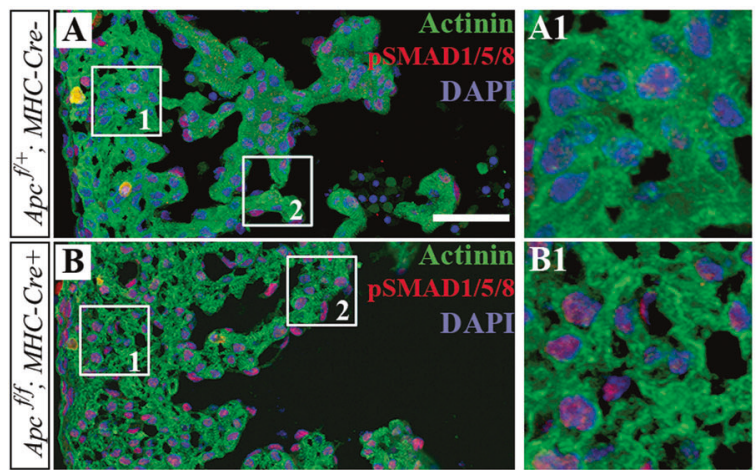

C
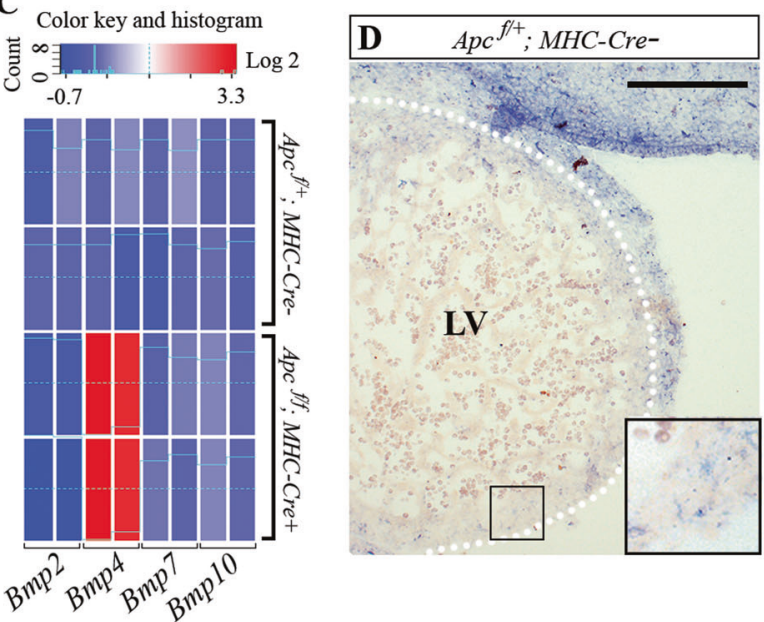
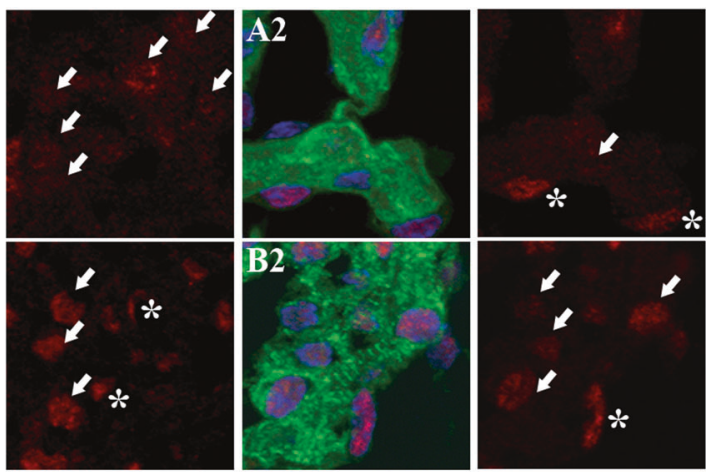

F

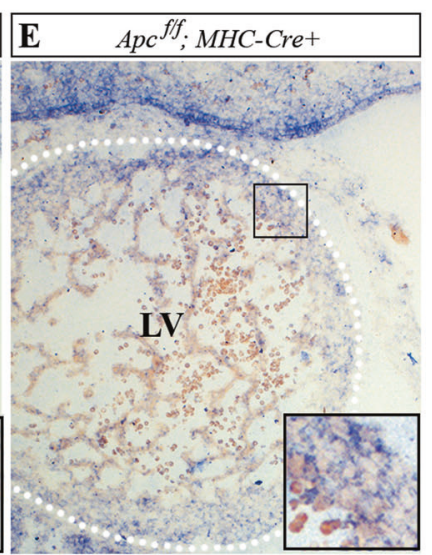

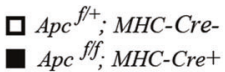

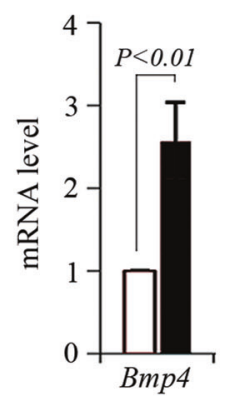

Fig. 4 Upregulation of BMP-SMAD1/5/8 signals in hearts by Apc deletion at E13.5 and E17.5. a, b pSMAD1/5/8 is upregulated in the cardiomyocytes (arrows) of Apc knockout animals (b) compared to their wild-type siblings (a) by immunofluorescent labeling. Noncardiomyocytes (asterisks) are also positive for $\mathrm{pSMAD} 1 / 5 / 8$, but no obvious changes are observed between wild-type and $A p c$ knockout hearts. c Apc deletion greatly increases Bmp4 mRNA level in E13.5 hearts, but has no effect on mRNA levels of Bmp2, Bmp7 and Bmp10. d, e Bmp4 in situ hybridization of E13.5 wild-type (d) and Apc deleted (e) hearts that were processed and imaged in parallel, showing an upregulation of Bmp4 mRNA level in the Apc knockout ventricles. Dotted lines indicate visceral pericardium. f At E17.5, cardiac Bmp4 mRNA remains consistently at high levels in Apc knockout mice. Scale bars $=50 \mu \mathrm{m}$. Data represent mean \pm SD. $N=3-4$ independent experiments cyclins are among the earliest identified canonical Wnt targets [29-32]. We previously demonstrated that only Cyclin D2 of three D-type cyclins was significantly increased in $A p c$ cKO hearts and decreased in $\beta$-catenin conditional knockout mice [3]. Similarly, Cyclin D2 is immediately induced by Apc loss-of-function in colon [32]. As shown in Fig. 4, Bmp4 expression was also significantly elevated by Wnt signaling activation resulting in Smad phosphorylation and activation. To determine whether Bmp4 and Cyclin D2 are direct targets of TCF/LEFs, SMADs, or both, we searched Bmp4 and Cyclin D2 promoters for TCF/LEF and SMAD consensus binding sites. There are three TCF/LEF sites and two SMAD sites in the Cyclin D2 promoter, and two TCF/LEF sites and one SMAD site in the Bmp4 promoter within $\sim 1600$ base pair upstream to $\sim 800$ base pair downstream from the transcriptional start site (Fig. 5a). Using ChIP and PCR with primers flanking these binding sites, we found that there was a switch from TCF7L2 to LEF1 and TCF7 in these sites with Wnt signaling activation in Apc deleted hearts. Additionally, we found that more $\beta$-catenin and acetylated histone $\mathrm{H} 4$ were present in these sites of Bmp4 and Cyclin D2 promoters in $A p c$ cKO hearts compared to WT controls (Fig. 5b, d). Interestingly, pSMAD1/5/8 were also increased in these sites, consistent with the notion that SMADs, TCF/ LEFs and $\beta$-catenin can form a tertiary complex in adjacent binding sites [33].

To further determine whether $T c f 7 l 2$ levels regulate Wnt target gene expression, we performed $T c f 7 l 2$ overexpression and knockdown in atrial-derived CMs (HL-1 cells). Similar to the heart with $\beta$-catenin deletion, increased expression of $T c f 7 l 2$ by infecting a full-length long form of $T c f 7 l 2$ in HL1 cells suppressed Bmp4 and Cyclin D2 expression. On the other hand, downregulation of Tcf7l2 by shRNA 
A

D2 Promoter region

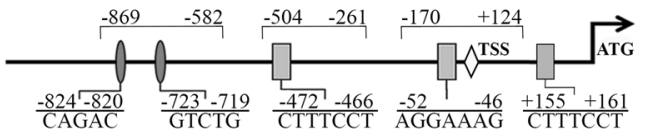

Bmp4 Promoter region

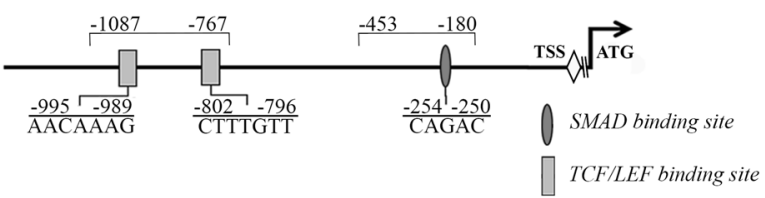

C

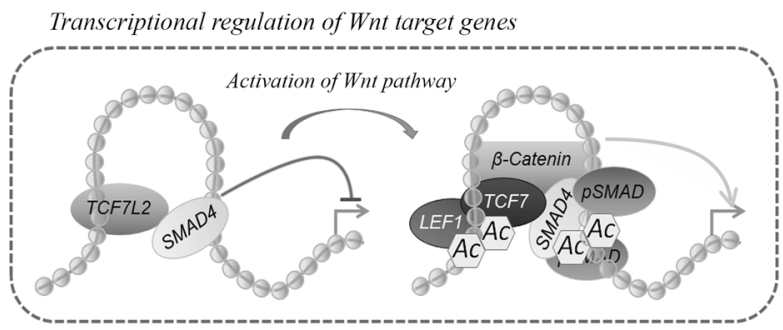

D
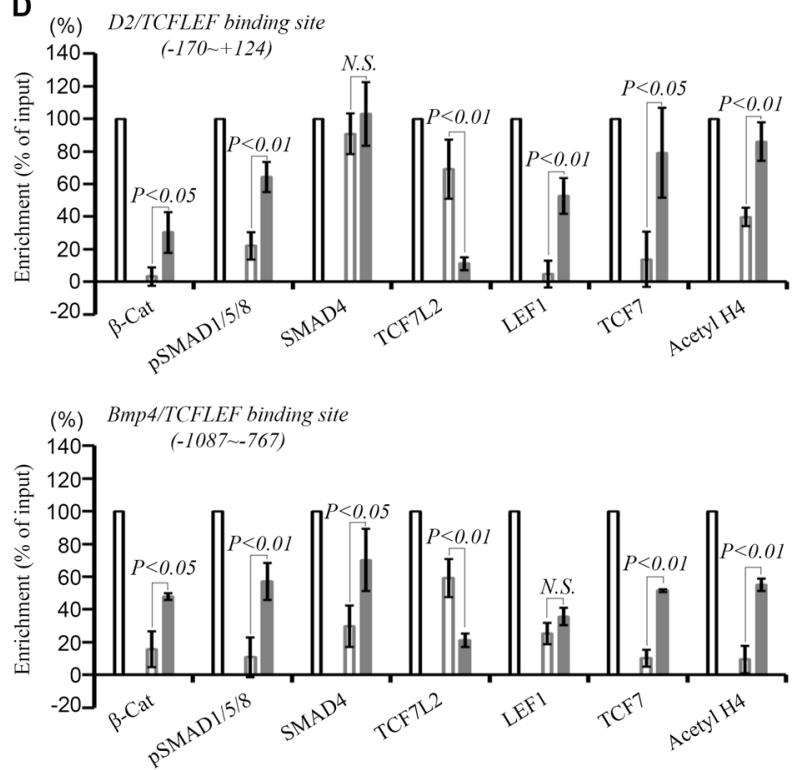

Fig. 5 Wnt nuclear effectors are recruited to the regulatory element of Cyclin D2 and Bmp4 promoters. a Amplicon positions and putative TCF/LEF and SMAD binding sites relative to transcription start site (TSS). b ChIP shows that the recruitments of $\beta$-catenin, SMAD4, pSMAD1/5/8, LEF1, and TCF7 to the TCF/LEF and SMAD binding sites in the Cyclin D2 and Bmp4 promoters are increased, while TCF7L2 is removed from these regions in Apc cKO compared to WT hearts at E17.5. These changes are accompanied by an increase in histone $\mathrm{H} 4$ acetylation (Acetyl $\mathrm{H} 4$ ), indicating transcriptional activation. Inputs are $2 \%$ of total mount chromatin. c A diagram shows Wnt

knockdown in HL-1 cells enhanced the expression of Lef1, Tcf7, Cyclin D2, and Bmp4 (Fig. 6). These results further confirm that TCF7L2 acts as a transcriptional suppressor in

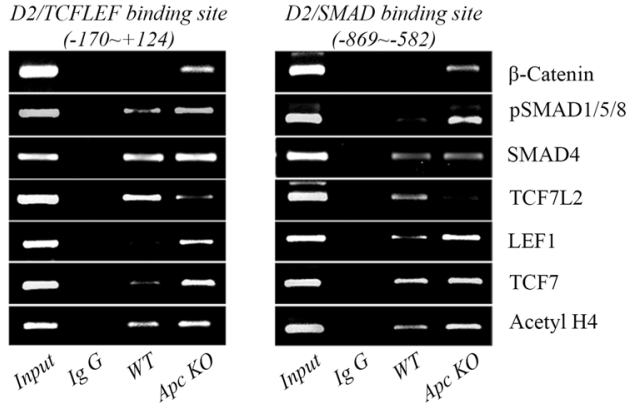

Bmp4/TCFLFF binding site

Bmp4/SMAD binding site
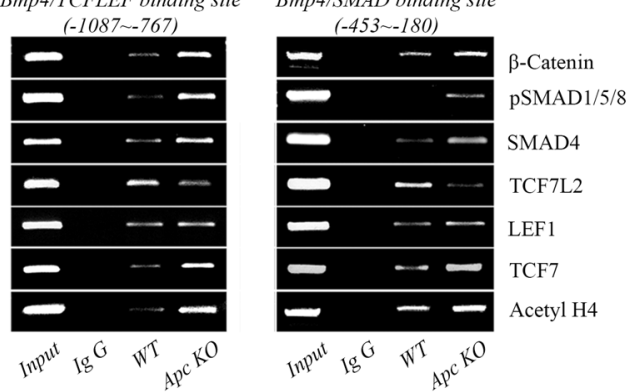

$\square$ Input

- $\operatorname{Ig} G$

$\square \mathrm{Apc}^{1+} ; \mathrm{MHC}-\mathrm{Cre}-$

$\mathrm{Apc}^{\mathrm{ff}} ; \mathrm{MHC}-\mathrm{Cre}+$
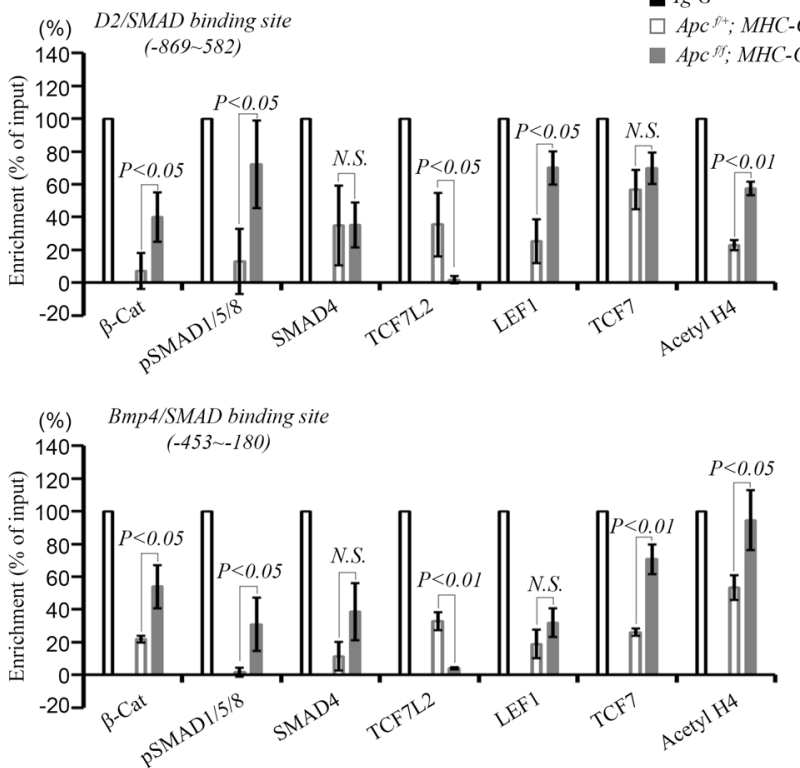

target gene regulation. In WT mice, TCF7L2 interacts with SMAD family members to suppress gene transcription. Upon Apc deletion, TCF7L2 is removed from TCF/LEF binding sites and replaced with activating forms of TCF/LEF family members, TCF7 and LEF1 to promote the recruitment of pSMAD1/5/8 and $\beta$-catenin as well as histone $\mathrm{H} 4$ acetylation. d Semiquantitative analysis of the relative enrichment of proteins bound to the TCF7L2 or SMAD consensus site. The intensity of each band in the gel images was measured using the ImageJ program and normalized by input. Each bar represents the mean $\pm \mathrm{SD}$ of four independent experiments

CMs and a switch of TCF7L2 with other activating TCF/ LEF family members, LEF1 and TCF7, is a novel mechanism for cardiac gene activation. 

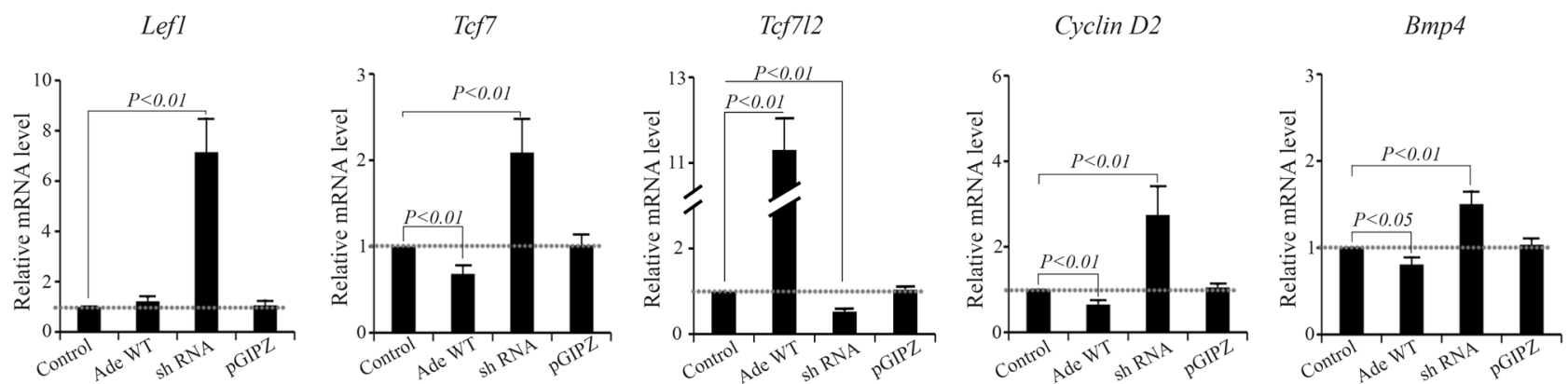

Fig. 6 Tcf7l2 levels control the expression of Lef1, Tcf7, Cyclin D2, and Bmp4. The mRNA levels of HL-1 CMs transfected with wild type long-form $T c f 7 l 2$ (Ade WT), $T c f 7 l 2$ shRNA (sh RNA), and control vector (pGPIZ) are quantified and compared with non-transfected group (Control) by real time qRT-PCR $72 \mathrm{~h}$ after transfection. $T c f 7 l 2$

\section{Discussion}

\section{Cardiac TCF/LEFs expression during heart development and upon Wnt activation}

LEF1 and TCF7 are widely expressed in mouse embryos at E7.5 and their expression coincides until day 10.5. Their expression patterns become more complex and only partially overlapping at later stages of murine development [34]. Using high-throughput instruments, Visel and colleagues have examined TCF/LEF expression patterns in E14.5 murine embryos by RNA in situ hybridization and showed that $T c f 7$, $T c f 7 l 1$, and $T c f 7 l 2$ mRNA are weakly detected in cardiac ventricles, but Lef1 mRNA has strong signal in E14.5 murine embryos. Herein, we showed that Lefl mRNA was mainly expressed in mesenchymal cells of developing heart valves and dramatically decreased from E13.5 to E17.5. Only scattered CMs demonstrated weak to moderate nuclear staining for LEF1 at E13.5, but not E17.5. Tcf7ll mRNA levels only showed minor change from E13.5 to E17.5. Tcf7 mRNA was very low relative to other three TCF/LEF members and decreased from E13.5 to E17.5. Among four TCF/LEF members, $T c f 7 l 2$ mRNA was the most abundant and showed only mild decrease from E13.5 to E17.5. Tcf7l2 splicing variants have been reported in different tissues, but the function of these variants remains undefined [9]. We found that $T c f 7 l 2$ without exon 4 increased from embryonic to neonatal hearts. Additionally, neonatal hearts have the most abundant $T c f 7 l 2$ transcripts with exon 14 .

By immunohistochemical staining, TCF7L1 was only detected with weak cytoplasmic, but not nuclear signals in CMs at E13.5, which became undetectable at E17.5. Mesothelial and endothelial cells, however, demonstrated nuclear positivity for TCF7L1. TCF7 protein was mainly detected in mesothelial and endothelial cells, but not in CMs by immunohistochemistry. Additionally, TCF7L2 was the only protein that could be detected in the nuclei of CMs at
shRNA significantly increases transcript levels of Lef1, Tcf7, Cyclin D2, and Bmp4. The mRNA levels of Bmp4 and Cyclin D2 are downregulated by $T c f 7 l 2$ overexpression. Data are presented as mean \pm SD from four independent experiments

E13.5 and E17.5 within the TCF/LEF family. These findings indicate that TCF7L2 is the main nuclear partner of $\beta$ catenin in CMs, especially during late stage of cardiogenesis. Moreover, nuclear TCF7L2 signal showed an increasing gradient from the epicardial to endocardial myocardium, which was in parallel to APC intensity gradient, but in contrast to $\beta$-catenin signals. This distribution pattern suggests that TCF7L2 may oppose Wnt/ $\beta$-catenin function as a suppressor of Wnt target genes.

\section{Positive crosstalk between Wnt/ $\beta$-Catenin and BMP- SMAD pathways}

The Canonical Wnt/ $\beta$-catenin pathway can cooperate with or antagonize BMP signaling $[11,12]$. Ablation of the $B m p$ receptor $1 a$ and $\beta$-catenin in early mesoderm progenitors by the MesP1-Cre causes similar cardiac defects [13]. Overexpression of a stabilized form of $\beta$-catenin enhances BMP production during early cardiac morphogenesis [13, 14]. Our results indicated that Wnt activation by cardiac $A p c$ deletion increased Bmp4 mRNA level. More importantly, we demonstrated that Smad 1/5/8, which are the immediate downstream molecules of BMP receptors and play a central role in BMP signal transduction, were phosphorylated and activated by $A p c$ deletion at E13.5 and E17.5. These findings suggest that there is a positive interaction of BMP signaling with the canonical $\mathrm{Wnt} / \beta$-catenin pathway in late heart development. In contrast, BMP and Wnt pathways have antagonistic roles in colon crypt development and maintenance [12].

\section{The switch of TCF7L2 with TCF7 and LEF1 in the regulatory elements of Wnt target genes enhances transcription}

Although all four TCF/LEF members can mediate the transcriptional activation of the reporter construct with 
multimerized TCF/LEF binding sites by $\beta$-catenin, they can be divided as either transcriptional activator or suppressor based on loss-of-function studies in intact organisms [5]. TCF7L1 generally suppresses Wnt target gene expression while LEF1 functions mainly as a transcriptional activator. On the other hand, TCF7L2 and TCF7 can suppress or activate gene expression dependent on cellular context and developmental stage. We found that TCF7L2 was the main $\mathrm{TCF} / \mathrm{LEF}$ member expressed in CMs of normal hearts from E13.5 to E17.5. The Wnt/ $\beta$-catenin activation in CMs by $A p c$ deletion increased LEF1 and TCF7 levels, but suppressed full length $75 \mathrm{kDa}$ TCF7L2 expression. More importantly, we revealed that TCF7L2 was switched off from the TCF/LEF binding sites in Bmp4 and Cyclin D2 promoters in exchange for TCF7 and LEF1. Furthermore, this switch was associated with increased $\beta$-catenin recruitment and histone $\mathrm{H} 4$ acetylation. Additionally, overexpression of Tcf7l2 suppressed Bmp4 and Cyclin D2 expression while lowering $T c f 7 l 2$ levels by shRNA knockdown enhanced Bmp4 and Cyclin D2 expression in HL-1 CMs. These data suggest that TCF7L2 is a Wnt suppressor in the heart similar to its role in intestines [35] and brain [36]. Genome-wide screening by RNA interference has demonstrated that TCF7L2 can suppress many Wnt responsive genes in colorectal cancer cells [10]. The switch of suppressive TCF7L1 and TCF7L2 for activating TCF7 and LEF1 has been considered as a general model for Wnt target gene activation [10, 37]. Our data confirm that this model of Wnt signal transduction also exists in the heart.

\section{Co-regulation of Wnt target genes by SMADs and TCF/LEFs}

SMAD-responsive element (SBE) is defined as 5'-CAGAC$3^{\prime}$. The affinity of Smad proteins for the SBE is too low to support binding of a Smad complex to a single SBE in vivo [38]. Many SMAD-responsive promoter regions with one or more SBEs still require cooperate with different DNA binding cofactor for effective DNA binding [39]. Adjacent SMAD and TCF/LEF binding sites are present in the regulatory elements of $M y c$, gastrin, and $M S x 2$ promoters [33, 40, 41]. SMADs and TCF/LEFs can be simultaneously recruited to these sites to form a regulatory complex. We identified multiple adjacent SMAD and TCF/LEF consensus sequences within $\sim 1600$ base pair upstream to $\sim 800$ base pair downstream from the transcriptional start site of Bmp4 and Cyclin D2 promoters (Fig. 4d). With Wnt activation upon $A p c$ deletion, the occupancy of SMAD4, pSMAD1/5/8, TCF7, LEF1, and $\beta$-catenin increased while TCF7L2 binding decreased in these sites. Our findings confirm the notion that SMADs, TCF/LEFs and $\beta$-catenin can form a tertiary complex in adjacent TCF/LEF and SMAD binding sites [33] and the cross-talk between Wnt and BMP signaling pathways is critical to the late stages of cardiac development.

The roles of canonical Wnt/ $\beta$-catenin signaling in cardiogenesis are dependent on the developmental stage and cellular context. The differential expression of LEF/TCF family members during different stages of heart development and in variety of heart diseases may determine the responsiveness of $\mathrm{CMs}$ to $\mathrm{Wnt} / \beta$-catenin activation and inhibition. More importantly, cell specific expression of these DNA binding transcriptional factors influences the output of $\mathrm{Wnt} / \beta$-catenin signaling in the heart as an intact organ. Our data reveal that Wnt signaling stimulates BMP4 production and coordinates with SMADs to promote CM proliferation during late fetal development. Therefore, the dysregulation of these pathways can lead to proliferation defect and cause congenital heart abnormalities.

Acknowledgements We thank Drs. Tetsuo Noda, Michael D. Schneider, and Bart O. Williams for their generosity to share mouse models with the scientific community. We also appreciate the technical support and data processing by Genomics Research Center of University of Rochester. The project described was supported by the National Institute of Health (NIH) grant R01 HL111480 (FL) and a Grant-in-Aid award 10GRNT4460014 (FL) from the American Heart Association Greater River Affiliate and the Lawrence J. and Florence A. DeGeorge Charitable Trust. HX is supported by the NIH R01 HL122793. YC and LL were supported by the NIH grant R01 HL136326.

\section{Compliance with ethical standards}

Conflict of interest The authors declare that they have no conflict of interest.

Publisher's note: Springer Nature remains neutral with regard to jurisdictional claims in published maps and institutional affiliations.

\section{References}

1. Hurlstone AF, Haramis AP, Wienholds E, Begthel H, Korving J, Van Eeden F, et al. The Wnt/beta-catenin pathway regulates cardiac valve formation. Nature. 2003;425:633-7.

2. Olson EN. Gene regulatory networks in the evolution and development of the heart. Science. 2006;313:1922-7.

3. Ye B, Hou N, Xiao L, Xu Y, Boyer J, Xu H, et al. APC controls asymmetric $\mathrm{Wnt} /$ beta-catenin signaling and cardiomyocyte proliferation gradient in the heart. $\mathrm{J}$ Mol Cell Cardiol. 2015;89:287-96.

4. Clevers H. Wnt/beta-catenin signaling in development and disease. Cell. 2006;127:469-80.

5. Cadigan KM, Waterman ML. TCF/LEFs and Wnt signaling in the nucleus. Cold Spring Harb Perspect Biol. 2012;4:a007906.

6. Arce L, Yokoyama NN, Waterman ML. Diversity of LEF/TCF action in development and disease. Oncogene. 2006;25:7492-504.

7. Merrill BJ, Pasolli HA, Polak L, Rendl M, Garcia-Garcia MJ, Anderson KV, et al. Tcf3: a transcriptional regulator of axis induction in the early embryo. Development. 2004;131:263-74.

8. Nguyen H, Merrill BJ, Polak L, Nikolova M, Rendl M, Shaver TM, et al. Tcf3 and Tcf4 are essential for long-term homeostasis of skin epithelia. Nat Genet. 2009;41:1068-75. 
9. Weise A, Bruser K, Elfert S, Wallmen B, Wittel Y, Wohrle S, et al. Alternative splicing of Tcf712 transcripts generates protein variants with differential promoter-binding and transcriptional activation properties at Wnt/beta-catenin targets. Nucleic Acids Res. 2010;38:1964-81.

10. Tang W, Dodge M, Gundapaneni D, Michnoff C, Roth M, Lum L. A genome-wide RNAi screen for Wnt/beta-catenin pathway components identifies unexpected roles for TCF transcription factors in cancer. Proc Natl Acad Sci USA. 2008;105:9697-702.

11. O'Connell DJ, Ho JW, Mammoto T, Turbe-Doan A, O'Connell JT, Haseley PS, et al. A Wnt-bmp feedback circuit controls intertissue signaling dynamics in tooth organogenesis. Sci Signal. 2012;5:ra4.

12. Reynolds A, Wharton N, Parris A, Mitchell E, Sobolewski A, Kam C, et al. Canonical Wnt signals combined with suppressed TGFbeta/BMP pathways promote renewal of the native human colonic epithelium. Gut. 2014;63:610-21.

13. Klaus A, Saga Y, Taketo MM, Tzahor E, Birchmeier W. Distinct roles of Wnt/beta-catenin and Bmp signaling during early cardiogenesis. Proc Natl Acad Sci USA. 2007;104:18531-6.

14. Kwon C, Arnold J, Hsiao EC, Taketo MM, Conklin BR, Srivastava D. Canonical Wnt signaling is a positive regulator of mammalian cardiac progenitors. Proc Natl Acad Sci USA. 2007;104:10894-9.

15. Shibata H, Toyama K, Shioya H, Ito M, Hirota M, Hasegawa S, et al. Rapid colorectal adenoma formation initiated by conditional targeting of the Apc gene. Science. 1997;278:120-3.

16. Cole AM, Myant K, Reed KR, Ridgway RA, Athineos D, Van den Brink GR, et al. Cyclin D2-cyclin-dependent kinase 4/6 is required for efficient proliferation and tumorigenesis following Apc loss. Cancer Res. 2010;70:8149-58.

17. Agah R, Frenkel PA, French BA, Michael LH, Overbeek PA, Schneider MD. Gene recombination in postmitotic cells. Targeted expression of Cre recombinase provokes cardiac-restricted, sitespecific rearrangement in adult ventricular muscle in vivo. J Clin Invest. 1997;100:169-79.

18. Muzumdar MD, Tasic B, Miyamichi K, Li L, Luo L. A global double-fluorescent Cre reporter mouse. Genesis. 2007;45:593-605.

19. Qu J, Zhou J, Yi XP, Dong B, Zheng H, Miller LM, et al. Cardiacspecific haploinsufficiency of beta-catenin attenuates cardiac hypertrophy but enhances fetal gene expression in response to aortic constriction. J Mol Cell Cardiol. 2007;43:319-26.

20. Yi XP, Gerdes AM, Li F. Myocyte redistribution of GRK2 and GRK5 in hypertensive, heart-failure-prone rats. Hypertension. 2002;39:1058-63.

21. Hill RE, Jones PF, Rees AR, Sime CM, Justice MJ, Copeland NG, et al. A new family of mouse homeo box-containing genes: molecular structure, chromosomal location, and developmental expression of Hox-7.1. Genes Dev. 1989;3:26-37.

22. St Amand TR, Zhang Y, Semina EV, Zhao X, Hu Y, Nguyen L, et al. Antagonistic signals between BMP4 and FGF8 define the expression of Pitx1 and Pitx2 in mouse tooth-forming anlage. Dev Biol. 2000;217:323-32.

23. Hou N, Ye B, Li X, Margulies KB, Xu H, Wang X, et al. Transcription factor 7-like 2 mediates canonical Wnt/beta-Catenin signaling and c-Myc upregulation in heart failure. Circ Heart Fail. 2016;9:e03010.

24. Travis A, Amsterdam A, Belanger C, Grosschedl R. LEF-1, a gene encoding a lymphoid-specific protein with an HMG domain, regulates T-cell receptor alpha enhancer function [corrected]. Genes Dev. 1991;5:880-94.
25. Visel A, Thaller C, Eichele G. GenePaint.org: an atlas of gene expression patterns in the mouse embryo. Nucleic Acids Res. 2004;32:D552-6.

26. Sedmera D, Reckova M, DeAlmeida A, Coppen SR, Kubalak SW, Gourdie RG, et al. Spatiotemporal pattern of commitment to slowed proliferation in the embryonic mouse heart indicates progressive differentiation of the cardiac conduction system. Anat Rec A Discov Mol Cell Evol Biol. 2003;274:773-7.

27. Ai D, Fu X, Wang J, Lu MF, Chen L, Baldini A, et al. Canonical Wnt signaling functions in second heart field to promote right ventricular growth. Proc Natl Acad Sci USA. 2007;104:9319-24.

28. Cohen ED, Wang Z, Lepore JJ, Lu MM, Taketo MM, Epstein DJ, et al. Wnt/beta-catenin signaling promotes expansion of Isl-1positive cardiac progenitor cells through regulation of FGF signaling. J Clin Invest. 2007;117:1794-804.

29. He TC, Sparks AB, Rago C, Hermeking H, Zawel L, da Costa LT, et al. Identification of c-MYC as a target of the APC pathway. Science. 1998;281:1509-12.

30. Shtutman M, Zhurinsky J, Simcha I, Albanese C, D'Amico M, Pestell R, et al. The cyclin D1 gene is a target of the beta-catenin/ LEF-1 pathway. Proc Natl Acad Sci USA. 1999;96:5522-7.

31. Tetsu O, McCormick F. Beta-catenin regulates expression of cyclin D1 in colon carcinoma cells. Nature. 1999;398:422-6.

32. Sansom OJ, Reed KR, Hayes AJ, Ireland H, Brinkmann H, Newton IP, et al. Loss of Apc in vivo immediately perturbs Wnt signaling, differentiation, and migration. Genes Dev. 2004;18:1385-90.

33. Hussein SM, Duff EK, Sirard C. Smad4 and beta-catenin co-activators functionally interact with lymphoid-enhancing factor to regulate graded expression of Msx2. J Biol Chem. 2003;278: 48805-14.

34. Oosterwegel M, van de Wetering M, Timmerman J, Kruisbeek A, Destree O, Meijlink F, et al. Differential expression of the HMG box factors TCF-1 and LEF-1 during murine embryogenesis. Development. 1993;118:439-48.

35. Angus-Hill ML, Elbert KM, Hidalgo J, Capecchi MR. T-cell factor 4 functions as a tumor suppressor whose disruption modulates colon cell proliferation and tumorigenesis. Proc Natl Acad Sci USA. 2011;108:4914-9.

36. Wang H, Matise MP. Tcf712/Tcf4 Transcriptional Repressor Function Requires HDAC Activity in the Developing Vertebrate CNS. PLoS ONE. 2016;11:e0163267.

37. Hikasa H, Sokol SY. Phosphorylation of TCF proteins by homeodomain-interacting protein kinase 2. J Biol Chem. 2011;286:12093-100.

38. Shi Y, Wang YF, Jayaraman L, Yang H, Massague J, Pavletich NP. Crystal structure of a Smad MH1 domain bound to DNA: insights on DNA binding in TGF-beta signaling. Cell. 1998;94:585-94.

39. Seoane J, Le HV, Shen L, Anderson SA, Massague J. Integration of Smad and forkhead pathways in the control of neuroepithelial and glioblastoma cell proliferation. Cell. 2004;117:211-23.

40. Lei S, Dubeykovskiy A, Chakladar A, Wojtukiewicz L, Wang TC. The murine gastrin promoter is synergistically activated by transforming growth factor-beta/Smad and Wnt signaling pathways. J Biol Chem. 2004;279:42492-502.

41. Hu MC, Rosenblum ND. Smad1, beta-catenin and Tcf4 associate in a molecular complex with the Myc promoter in dysplastic renal tissue and cooperate to control Myc transcription. Development. 2005; 132:215-25. 